\title{
Sudoku löst epileptischen Anfall aus
}

\begin{abstract}
Ein Mann entwickelt nach einem überstandenen Lawinenunglück plötzlich klonische Anfälle. Diese treten aber nur dann auf, wenn er Sudokus löst. Verzichtet er darauf, hat er keine Probleme.
\end{abstract}

Der 25-Jährige hatte Glück im Unglück: Während einer Skitour wurde er von einer Lawine begraben und konnte bereits nach 15 Minuten befreit werden. Allerdings bekam er in dieser Zeit zu wenig Luft und entwickelte posthypoxische Myoklonien, von denen vor allem der Mund beim Sprechen und die Beine beim Gehen betroffen waren, nicht aber die Arme, berichten Ärzte um Privatdozent Berend Feddersen von der Klinik und Poliklinik für Palliativmedizin der LMU München. Als der Patient einige Wochen später versuchte, ein Sudoku-Rätsel zu lösen, fing jedoch plötzlich der linke Arm an, heftig zu zucken. Die klonischen Anfälle traten immer nur dann auf, wenn er sich mit Sudokus beschäftigte, nicht aber bei anderen kognitiven Tätigkeiten wie Lesen, Schreiben oder Rechnen. Die Anfälle hörten auch sofort wieder auf, wenn er die Rätsel zur Seite legte.

\section{Verlust von U-Fasern führte zu Übererregbarkeit}

Im EEG zeigte sich beim Versuch, ein Sudoku zu lösen, ein rechtsseitiges, zentroparietales Anfallsmuster. Der Mann versuchte dabei stets, sich die Zahlenreihen dreidimensional vorzustellen. Wurden ihm nun ähnliche räumlich-visuelle Aufgaben gestellt, etwa Zahlen nach einem bestimmten Muster zu ordnen, führte dies ebenfalls zu den bekannten Anfällen. Im MRT fanden die Ärzte keine Auffälligkeiten. Sie versuchten es daher mit einem Technetium-PET. Dabei fanden sie eine Hyperperfusion der Pars posterior des Gyrus cinguli. Im fMRT zeigte sich während der Beschäftigung mit Sudokus eine breit gefächerte Aktivität vor allem im zentroparietalen Kortex. In dieses Bild passt ein rechtsseitiger, zentroparietaler Mangel an inhibitorischen U-Fasern, wie er sich per Diffusions-Tensor-Bildgebung (DTI) fest- stellen ließ. Bestätigt wurde die Hyperexzitabilität dieses Areals zudem durch somatosensibel evozierte Potenziale am linken Mittelarmnerv.

Die plausibelste Erklärung für die Sudoku-induzierten Anfälle: Während der Mann unter den Schneemassen nach Luft rang, kam es sowohl zu einer verbreiteten diffusen Schädigung im Gehirn als auch zu einem lokalen Verlust von rechtsseitigen zentroparietalen U-Fasern, schreiben Feddersen und Mitarbeiter. Dieser Verlust führte zu einer Übererregbarkeit im zentroparietalen Kortex. Komplexe räumlichvisuelle Aufgaben, die zu einer Aktivierung dieses Bereichs führen, genügen nun, um einen fokalen Anfall auszulösen. Ähnliche Formen von Reflex-Epilepsien, getriggert durch z.B. Kopfrechnen, den Versuch, Probleme zu lösen, oder bei bestimmten

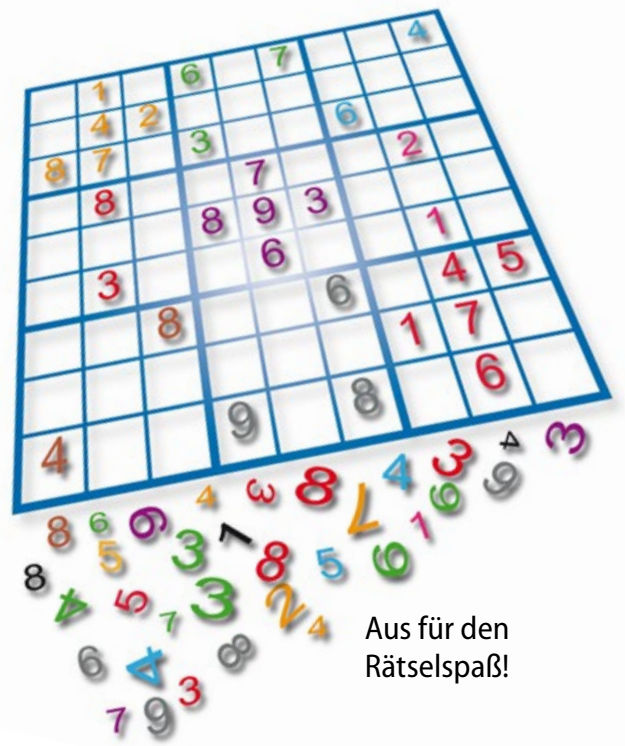

Spielen, wurden in der Literatur immer wieder beschrieben, so Feddersen. Allerdings handelte es sich dabei fast immer um Patienten mit idiopathischer Epilepsie. Bei dem Lawinenopfer war hingegen keine antiepileptische Langzeittherapie nötig. Er durfte nur keine Sudokus mehr lösen. Daran hat er sich dann auch gehalten und ist seit nunmehr fünf Jahren anfallsfrei. (mut)

Feddersen B et al. Seizures From Solving Sudoku

Puzzles. JAMA Neurol. ePub 19.10.2015

\section{„Innere Uhr" wird korrigiert}

\section{Lichttherapie: Nicht nur gegen Winterdepression}

Die stimmungsaufhellende Wirkung einer Lichttherapie beschränkt sich nicht auf Herbst-Winter-Depressionen. Die Behandlung ist auch bei nichtsaisonaler Major-Depression wirksamer als eine Scheintherapie.

Kanadische Psychiater haben die Wirkung einer Lichttherapie in einer Studie überprüft. An der Studie waren 122 Patienten im Alter von 19 bis 60 Jahren mit mindestens mittelschwerer Depression beteiligt. Sie wurden acht Wochen lang mit Licht plus Placebotablette (32 Patienten), mit Fluoxetin $20 \mathrm{mg} / \mathrm{d}$ plus Scheingerätetherapie (31 Patienten), mit Licht plus Fluoxetin (29 Patienten) oder nur mit Placebotherapien (20 Patienten) behandelt. Primärer Studienendpunkt war die Verbesserung der depressiven Symptomatik gemäß der Montgomery-Åsberg-Depression-RatingSkala (MADRS, 0-60 Punkte). Der MADRS-Wert sank mit der Lichttherapie im Mittel um 13,4, mit Fluoxetin um 8,8, mit der Kombination um 16,9 und mit einer reinen Placebotherapie um 6,5 Punkte. Nur mit Licht sowie mit der Kombinationstherapie war der Unterschied zur Placebotherapie signifikant. Die Kombination aus Licht und SSRI war zudem auch der alleinigen SSRI-Therapie überlegen. Ein Therapieansprechen, sprich eine Reduktion des anfänglichen MADRS-Scores um mindestens $50 \%$, zeigten 50,0\% (Licht), 29,0\% (SSRI), 75,9\% (Licht + SSRI) und 33,3\% der Patienten (Placebo).

(bs)

Lam RW et al. JAMA Psychiatry 2015, epub 18.11 .15 